\title{
Model for humpback relaxation oscillations
}

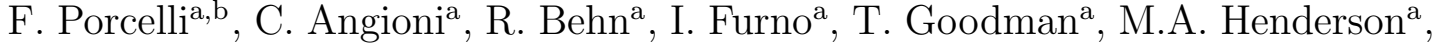

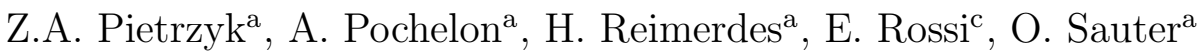 \\ ${ }^{a}$ Centre de Recherches en Physique des Plasmas, Association Euratom-Confédération Suisse, \\ Ecole Polytechnique Fédérale de Lausanne, Lausanne, Switzerland \\ ${ }^{\text {b }}$ Istituto Nazionale Fisica della Materia and Dipartimento di Energetica, \\ Politecnico di Torino, Turin, Italy \\ ${ }^{c}$ Institute for Fusion Studies, University of Texas at Austin, \\ Austin, Texas, United States of America
}

\begin{abstract}
An interpretation of non-standard, central MHD events in the TCV tokamak during localized ECRH is presented. It is shown that the non-standard behaviour is a consequence of specific features in the electron temperature profile produced when ECRH power is deposited close to the $q=1$ surface and by the advection and mixing of electron thermal energy resulting from a resistive MHD instability.
\end{abstract}

Experimental research on controlled thermonuclear fusion requires the production of hot plasmas with temperatures in the multi-keV range. One effective scheme providing intense localized heating is ECRH [1]. Resonant electromagnetic waves are launched into a magnetically confined plasma at a frequency matching a low integer multiple of the local EC frequency, $\omega_{c e}=e B / m c$. In recent years, record values of ECRH power density have been attained. This has revealed new and peculiar plasma behaviour. One example is the observation of multipeaked electron temperature profiles and sharp temperature gradients in the RTP [2] and TEXT Upgrade [3] experiments, explained in Ref. [4] as being the consequence of interaction between ECRH and resistive MHD modes with toroidal $n=1$ and dominant poloidal $m=1$ mode numbers. These modes are known to be responsible for the well known internal plasma sawtooth relaxation oscillations [5]. In TCV experiments with intense ECRH, sawtooth oscillations acquire a non-standard character, as reported in Refs $[6,7]$. In particular, when the ECRH power is deposited close to the $q=1$ surface, the line integrated soft $\mathrm{X}$ ray temporal traces exhibit peculiar shapes, which suggested the nickname 'humpback' when they were first observed in T-10 experiments [8]. The purpose of this Letter is to propose a theoretical interpretation of the humpback phenomenon. We argue that specific features in the electron temperature profile are produced by localized ECRH. Then, the humpback behaviour is consistent with the advection and mixing of electron thermal energy in the plasma core associated with the excitation of resistive $m / n=1$ internal modes.

TCV is a tokamak with major radius $R=0.88 \mathrm{~m}$, minor radius $a=0.25 \mathrm{~m}$, vacuum vessel elongation $\kappa=3$ and central magnetic field $B=1.43 \mathrm{~T}$ in vacuum. Up to three $82.7 \mathrm{GHz}, 500 \mathrm{~kW}$ gyrotrons, each with a pulse length of $2 \mathrm{~s}$, for heating at the cyclotron second harmonic resonance via the extraordinary mode, were used in this study. The vertical microwave beam diameter near the plasma centre is of the order of $5 \mathrm{~cm}$, while the horizontal width of the deposition region is $O(1 \mathrm{~cm})$. ECRH power densities in excess of $1 \times 10^{2} \mathrm{MW} / \mathrm{m}^{3}$ can be obtained. New insight into the temporal evolution of the different types of MHD modes observed in TCV has become possible because of the recently upgraded, 200 channel soft X ray tomographic system, allowing for a sampling time of $13 \mu \mathrm{s}$. The spatial reconstruction of the central emissivity profile is based on a pixel method with characteristic spatial resolution of about $3 \mathrm{~cm}$, to be compared with a typical sawtooth inversion radius of $5-10 \mathrm{~cm}$.

Figure 1 compares standard and humpback relaxation oscillations in TCV. Standard sawteeth, shown in Figs 1(a,b), are observed with auxiliary heating when the ECRH power is deposited on the magnetic axis. Figure 1(a) presents the line integrated soft $\mathrm{X}$ ray intensity, $I_{X}$, for a vertical chord through the plasma centre as a function of time and Fig. 1(b) presents a tomographic reconstruction of the soft $\mathrm{X}$ ray emissivity isocontours. 


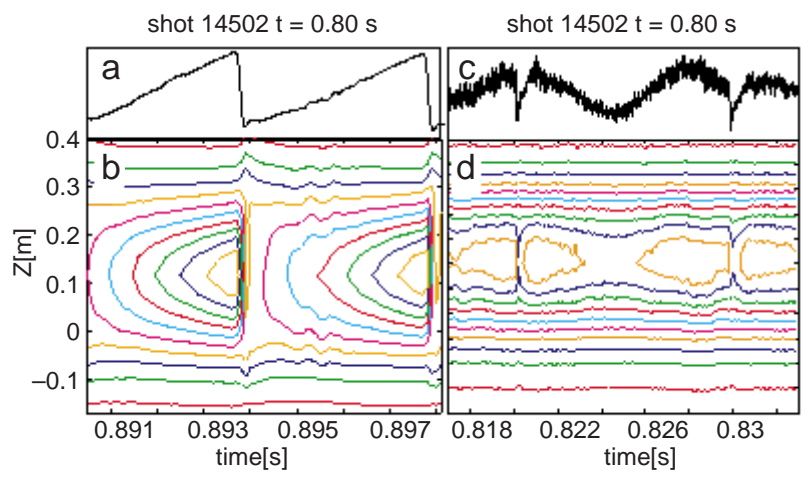

Figure 1. Standard (a,b) and non-standard (c,d) central MHD activity in TCV. Frames (a) and (c), central soft $\mathrm{X}$ ray line integrated intensity as a function of time; (b) and $(\mathrm{d})$, soft $\mathrm{X}$ ray emissivity isocontours.

With off-axis ECRH, different types of nonstandard oscillations are observed [6]. For instance, so-called 'saturated sawteeth' are often observed when the wave energy is deposited within the magnetic surface where the magnetic winding index (safety factor), $q$, equals unity. Humpback relaxations, which are the focus of this Letter, are observed when the ECRH power is deposited close to the $q=1$ surface. The resulting humpback behaviour for the line integrated soft $\mathrm{X}$ ray traces is shown in Fig. 1(c). In Fig. 1(d) we show the corresponding emissivity isocontours. Humpback relaxation oscillations were also observed in the T-10 tokamak with central electron cyclotron (counter-)current drive [8]. However, current drive is not an essential ingredient for the appearance of humpbacks in TCV.

The distinctive feature of humpback relaxations is the fast drop and rise of the central soft $\mathrm{X}$ ray emissivity, on a timescale normally below $1 \mathrm{~ms}$ in TCV. We show later that the relaxation process, including the fast rise, is associated with the development of the $m / n=1$ instability. After the fast events, a diffusive and heating phase follows, during which central emissivity is again observed to drop and rise, but on a slow timescale. The overall change in intensity is much smaller for a humpback than for a standard sawtooth for comparable ECRH power, safety factor and total plasma energy content. For instance, in Fig. 1, $\Delta I_{x} \sim 8 \%$ for the humpback and $\sim 25 \%$ for the standard sawtooth. The repetition time for humpback relaxations can be significantly longer than that for the standard sawtooth period.

One way to analyse soft $\mathrm{X}$ ray data is the singular value decomposition (SVD) method [9]. The inverted emissivity measurements form a signal

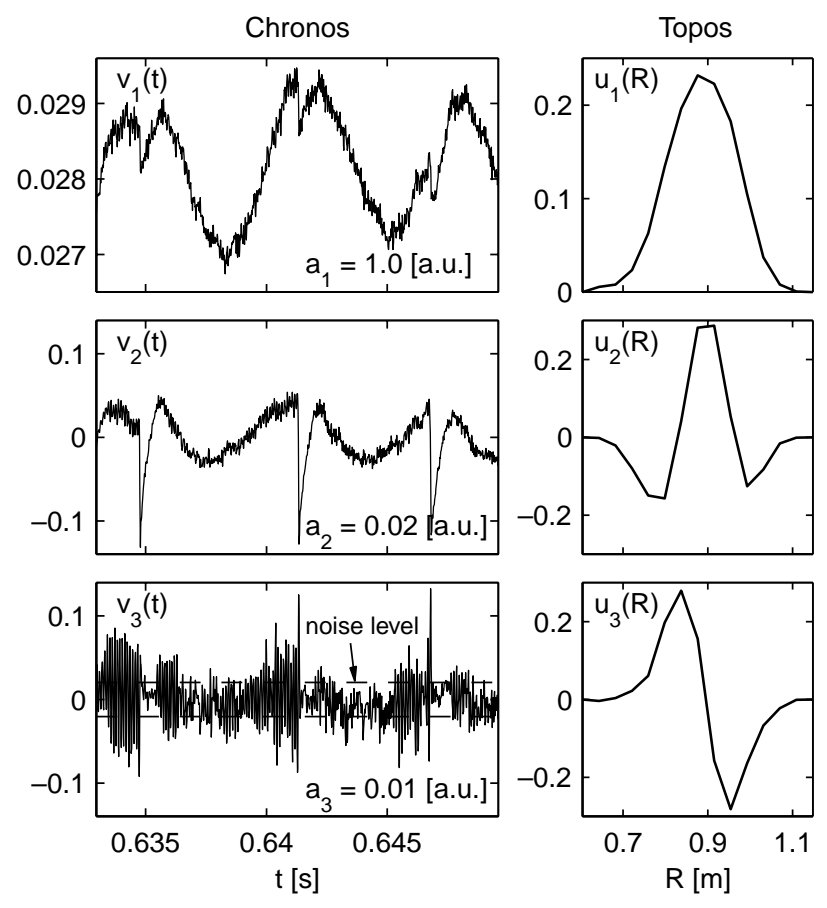

Figure 2. Singular value decomposition of soft $\mathrm{X}$ ray emissivity for the case of a humpback oscillation.

matrix, $X$, which is decomposed into orthonormal spatial eigenvectors $u$ 'topos' and temporal eigenvectors $v$ 'chronos', resulting in a separation of variables $X(x, t)=\sum a_{k} u_{k}(x) v_{k}(t)$. The three dominant pairs of eigenvectors characterizing a humpback oscillation are shown in Fig. 2. The $k=1$ amplitude, $a_{1}$, is the largest by two orders of magnitude. For simplicity, only a horizontal cut through the plasma centre of the topos is shown. These can be interpreted as follows: $u_{1}$, average emission profile; $u_{2}$, peaking or flattening of the average profile; $u_{3}, m=1$ structure of the emissivity profile. The $k=1$ chrono oscillates on the timescale of the humpback period. Between subsequent relaxations, the amplitude of the $k=2$ chrono is nearly constant as compared with its variation during the relaxations, which indicates that the $\mathrm{X}$ ray emissivity does not show the typical peaking of standard sawteeth, but remains nearly flat within the $q=1$ region. This is in contrast with standard sawteeth, which usually exhibit a monotonic increase of the $k=2$ amplitude, indicating gradual peaking between subsequent relaxations. The third pair of eigenvectors shows an $m=1$ oscillation which significantly exceeds the estimated noise level just before the relaxation phase. The different time behaviours of the three eigenvectors suggest that the fast drop and rise of the relaxation phase is associated with 
MHD activity, while the slow oscillation phase is a global phenomenon of the emissivity profile which can be ascribed to a transport process.

Our understanding of the humpback phenomenon, in particular of the fast drop and rise behaviour of the soft $\mathrm{X}$ ray emissivity, is based on the following heuristic picture. When the ECRH power is deposited near the $q=1$ radius, $r_{1}$, the temperature profile tends to acquire distinctive features. Specifically, the electron temperature can become relatively flat, perhaps even hollow, in the central region up to the $q=1$ radius and relatively steep outside this radius, as the result of localized heating and diffusive transport during periods of relative MHD quiescence. These periods are terminated by the onset of $m / n=1$ magnetic islands $[10,11]$. Since the formation of these specific features in the electron temperature profile requires relatively long quiescent periods, improved stability against $m / n=1$ modes is an important factor. This improvement is possible because ECRH power deposited near the $q=1$ surface can lead to a reduction of the magnetic shear near that surface (via an increase of the local electrical conductivity), which is known from experiments [12-14] and theory [15, 16] to produce a stabilizing influence.

Now, let us indicate with $T_{0}, T_{1}$ and $T_{m i x}$ the values of the electron temperature before the onset of the $m / n=1$ magnetic island, on the magnetic axis, at the $q=1$ radius and at the sawtooth mixing radius [10] $r_{m i x}$, respectively. For the case of a humpback, $T_{0} \sim T_{1}>T_{m i x}$, while for a standard sawtooth $T_{0}>T_{1}>T_{m i x}$. The $m / n=1$ magnetic island can grow on a timescale faster than the heating and diffusion timescales. Thus, during the island growth, the temperature on the displaced magnetic axis remains nearly constant, i.e. close to $T_{0}$. Similarly, the temperature at the island $\mathrm{O}$ point, which corresponds to the flux surface of radius $r_{1}$ before the onset of the island, remains close to $T_{1}$. Figures $3(\mathrm{a}, \mathrm{b})$ illustrate a humpback relaxation event. Figure 3(a) shows the situation when the displacement, $\xi$, of the original magnetic axis is about $0.5 r_{m i x}$. Since $T_{0} \sim T_{1}$, both the plasma core and the island $\mathrm{O}$ point region are relatively hot. Between these two peaks, a valley in the temperature profile is produced, with a minimum value, $T_{m i n}$, such that $T_{1}>T_{m i n} \geq T_{m i x}$. In fact, the inner and outer legs of the island separatrix are in thermal contact, as they are part of the same flux surface cross-section, so that magnetic field lines on or near the separatrix connect central plasma regions with regions at a radial distance between $r_{1}$ and $r_{m i x}$ from the centre. This valley moves rapidly across the

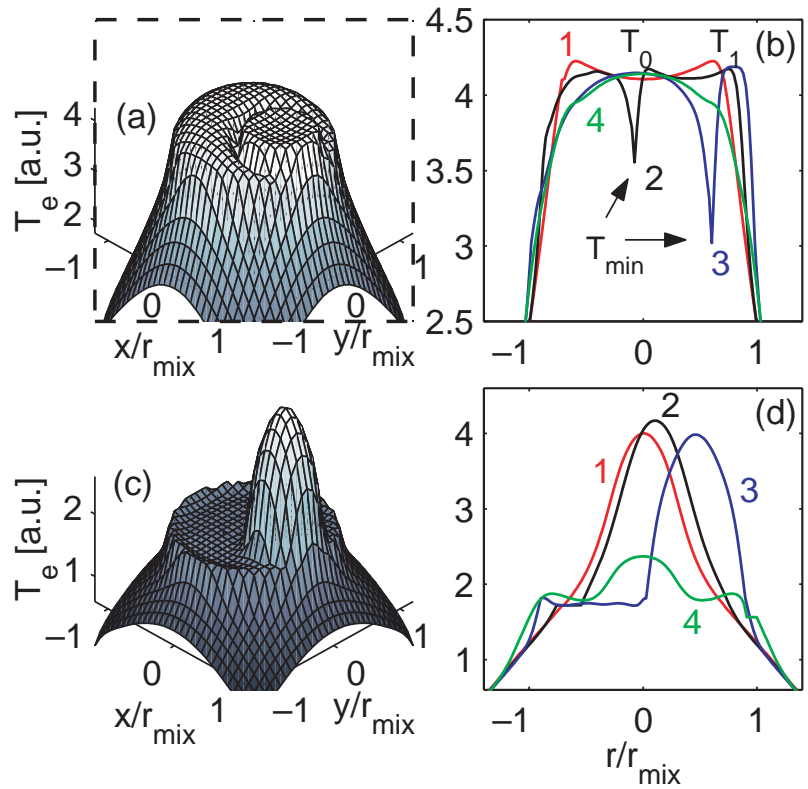

Figure 3. Simulation results. Humpback relaxation illustrated by (a) the temperature profile for a value of the displacement function normalized to the mixing radius, $\xi=0.53$, and (b) temperature profile sections through the island $\mathrm{X}$ and $\mathrm{O}$ points at $\xi=0.14$ (curve 1), 0.53 (curve 2), 0.9 (curve 3) and 1.0 (curve 4). Standard sawtooth, illustrated by (c) the temperature profile at $\xi=0.62$ and $(\mathrm{d})$ sections at $\xi=0.01$ (curve 1 ), 0.15 (curve 2), 0.62 (curve 3) and 0.95 (curve 4).

plasma centre on the timescale of the island growth, as shown in Fig. 3(b). Thus, the plasma temperature at a fixed central point drops and rises quickly during the passage of this valley, reproducing the fast drop and rise relaxation phase of a humpback. Note that, starting from a slightly hollow temperature profile peaked at $r_{1}$ (curve 1 of Fig. 3(b)), the relaxed profile is peaked on-axis (curve 4), as the reconnection process in this case entails a net convection of thermal energy from the $q=1$ surface to the centre. Then, a slow diffusive phase follows, during which the electron temperature on-axis slightly drops and recovers as ECRH power is deposited off-axis.

The case of a standard sawtooth is contrasted in Figs $3(\mathrm{c}, \mathrm{d})$. In this case, the hot core is displaced while maintaining a temperature close to $T_{0}$, while the island region is relatively cold, with a temperature about $T_{1}$ intermediate between $T_{0}$ and $T_{m i x}$.

Figure 3 is actually the result of a quantitative simulation model, first proposed in Ref. [4], which solves the thermal energy diffusion equation taking into account the anisotropy introduced by the 

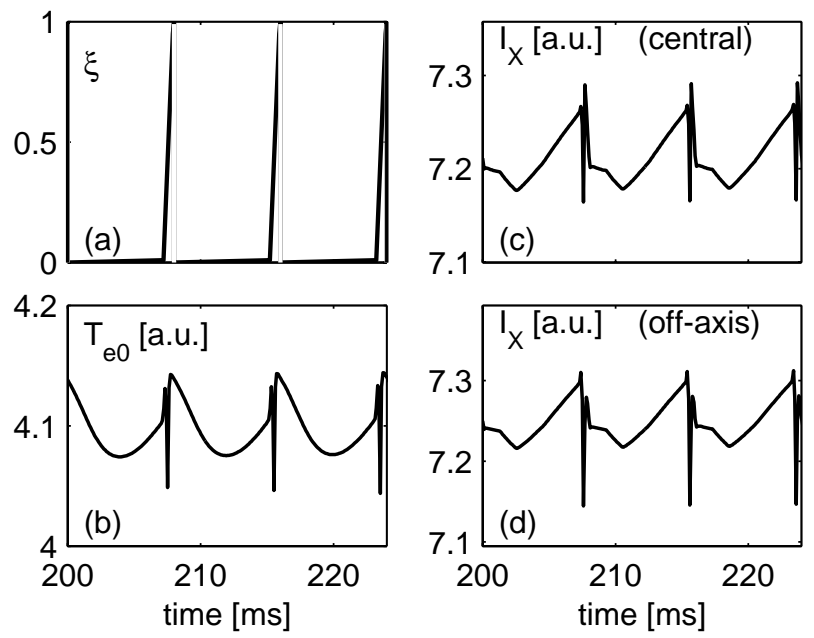

Figure 4. Simulation results, showing three periods of a humpback relaxation oscillation: (a) the assumed displacement function $\xi(t)$, (b) central temperature evolution, (c) central line integrated soft $\mathrm{X}$ ray trace, (d) off-axis line integrated soft $\mathrm{X}$ ray trace.

magnetic field (heat diffusion along magnetic field lines is much faster than cross-field diffusion), a localized electron heat source, plasma rotation and a growing $m / n=1$ magnetic island. The $m / n=1$ magnetic topology is described by a helical flux function, $\psi_{\star}=\psi_{\star}(r, \alpha, \xi)$, where $\alpha=\theta-\phi$ and $\xi(t)$ is the radial displacement of the hot core magnetic axis as a function of time. Figure 2 of Ref. [4] illustrates a poloidal cross-section of the $\psi_{\star}=$ const flux surfaces and the ECRH heating region. As a consequence of plasma rotation, the ECRH power is effectively deposited within the annular region between the radii $r_{h 1}$ and $r_{h 2}$ on the poloidal midplane. A diffusion equation describing the evolution of the temperature on magnetic flux tubes frozen to the plasma flow is introduced. At the particular instant in time when two flux surfaces with equal helical flux reconnect, a mixing rule for the thermal energy is implemented [4]. In our model, the function $\xi(t)$ is a free parameter not determined theoretically. Reasonable trial functions for $\xi(t)$ are suggested by stability considerations and may be inferred from experimental data.

Typical simulation results for humpback oscillations are shown in Figs 3(a,b) and 4. The input parameters are $r_{h 1} / r_{1}=0.85, r_{h 2} / r_{1}=1.15$, $\chi_{\perp} \tau_{\text {saw }} / r_{1}^{2}=0.2$ and $P_{E C} \tau_{\text {saw }} /\left(V_{1} n\right)=1.9 \mathrm{keV}$, where $\chi_{\perp}$ is the cross-field thermal energy diffusion coefficient, assumed to be constant for $r \leq r_{1}$, $\tau_{\text {saw }}$ is the oscillation period, $P_{E C}$ is the ECRH coupled power and $V_{1}=2 \pi^{2} R r_{1}{ }^{2}$. For instance, with $\tau_{\text {saw }}=3 \mathrm{~ms}, r_{1}=10 \mathrm{~cm}$ and $n=3 \times 10^{19} \mathrm{~m}^{-3}$, one obtains $\chi_{\perp}=0.7 \mathrm{~m}^{2} / \mathrm{s}$, a realistic value within the $q=1$ radius, and $P_{E C}=500 \mathrm{~kW}$. The function $\xi(t)$ used in this simulation is shown in Fig. 4(a). Figure 4(b) shows the central electron temperature as a function of time. In order to obtain a humpback with our simulation code, one needs to assume heating near the $q=1$ radius and a relatively long period between successive relaxations during which $\xi(t) \approx 0$, consistent with the experimental indications, so that during the quiescent periods the electron temperature can build up in the region where the heating power is concentrated and $T_{1}$ can become comparable to or even larger than $T_{0}$. Figures $4(\mathrm{c}, \mathrm{d})$ show simulated line integrated soft $\mathrm{X}$ ray traces for two different vertical viewing chords: Fig. 4(c) is from a chord through the centre and Fig. 4(d) is from a chord at a distance $0.4 r_{1}$ from the centre. The detailed shapes of the line integrated traces depend on the viewing chord.

The simulations in Figs 3 and 4 give a fast drop and rise of the local electron temperature occurring on the same timescale. However, with a more structured functional form for $\xi(t)$, the fast rise can be made slower than the fast drop. This may be important for a more detailed comparison with the experimental traces, as will be shown in a later publication.

For markedly different choices of parameters, different types of non-standard sawtooth traces can be obtained. In particular, if the ECRH power is deposited well within the $q=1$ region, we can reproduce saturated as well as triangular sawteeth, depending on the choice of $\xi(t)$. An example of a simulated saturated sawtooth was shown in Ref. [7]. Multipeaked temperature profiles of the type observed in RTP [2] are obtained with our code assuming narrower ECRH deposition widths and a magnetic island which grows slowly during a large fraction of the time between relaxations.

In conclusion, we have discussed a new type of non-standard relaxation oscillations observed in ECRH plasmas produced by TCV and T-10, the socalled humpbacks. The explanation of the humpback phenomenon, as first proposed in this Letter, requires specific features of the electron temperature profile produced by localized ECRH and a fairly standard model for $m / n=1$ magnetic islands. Indeed, in our model, the evolution of these islands obeys the topological constraints of the Kadomtsev model [10]. However, the humpback phenomenon was unforeseen and could be brought to light only with localized 
electron heating. More specifically, we have shown that intense localized ECRH near the $q=1$ radius can produce temperature profiles which are nearly flat up to the $q=1$ radius and which fall steeply beyond that radius. The fast drop and rise of the soft $\mathrm{X}$ ray line integrated intensity for a humpback is then shown to follow the development of an $m / n=1$ magnetic island and the corresponding advection and mixing of thermal energy.

The essential aspects of this behaviour are captured by our model. The model is also able to reproduce different types of non-standard sawteeth, such as saturated sawteeth, as shown in Ref. [7]. However, the model is admittedly very rough and there are many open issues that call for extensions. One issue relates to current drive effects, which probably play an important role for the detailed interpretation of humpback events observed [8] in T-10. Since current drive modifies the $q$ profile and the local magnetic shear, we expect this to have consequences for $m / n=1$ mode stability which could be taken into account by different choices of the displacement function in our simulation code. Double tearing modes excited by non-monotonic $q$ profiles may give rise to a similar relaxation phenomenology and could play a role in the understanding of non-standard relaxation traces sometimes observed when ECRH is deposited close to the $q=2$ surface. Another open issue is the overall detailed shape of a humpback trace. In particular, the slow drop and rise of the soft X ray emissivity between successive relaxations is most likely the result of transport. Our simulations with constant density and $\chi_{\perp}$ do show a tendency for central electron temperature to slowly drop and rise between humpback relaxations; however, more accurate modelling requires realistic transport coefficients for the plasma. The simulation results support our heuristic picture of the humpback event and are in good qualitative agreement with the experimental traces. These results are relatively insensitive to small variations of the model input parameters and of the function $\xi(t)$.

\section{Acknowledgements}

The authors are grateful to K.A. Razumova and H. Weisen for useful discussions. This work was supported in part by the Swiss National Science Foundation and by the Italian National Research Council (CNR).

\section{References}

[1] Lloyd, B., Plasma Phys. Control. Fusion 40 (1998) A119.

[2] Lopes Cardozo, N.J., et al., Phys. Rev. Lett. 73 (1994) 256.

[3] Cima, G., et al., Plasma Phys. Control. Fusion 40 (1998) 1149.

[4] Porcelli, F., et al., Phys. Rev. Lett. 82 (1999) 1458.

[5] von Goeler, S., et al., Phys. Rev. Lett. 33 (1974) 1201.

[6] Pietrzyk, Z.A., et al., Nucl. Fusion 39 (1999) 587.

[7] Pochelon, A., et al., Nucl. Fusion 39 (1999) 1807.

[8] Razumova, K.A., et al., Plasma Phys. Rep. 23 (1997) 13.

[9] Dudok de Wit, T., et al., Phys. Plasmas 1 (1994) 3288.

[10] Kadomtsev, B.B., Fiz. Plazmy 1 (1975) 710 [English translation: Sov. J. Plasma Phys. 1 (1975) 389].

[11] Coppi, B., et al., Fiz. Plazmy 2 (1976) 961 [English translation: Sov. J. Plasma Phys. 2 (1976) 533].

[12] Snider, R.T., et al., Phys. Fluids B 1 (1989) 404.

[13] Levington, F.M., et al., Phys. Rev. Lett. 72 (1994) 2895.

[14] Bhatnagar, V.P., et al., Nucl. Fusion 34 (1994) 1579.

[15] Porcelli, F., et al., Plasma Phys. Control. Fusion 38 (1996) 2163.

[16] Sauter, O., et al., in Theory of Fusion Plasmas (Proc. Joint Varenna-Lausanne Int. Workshop, Varenna, 1998), Editrice Compositori, Bologna (1999).

(Letter received 10 January 2000

Final manuscript accepted 18 July 2000)

E-mail address of F. Porcelli: porcelli@polito.it

Subject classification: B0, Te; G0, Ti 